\title{
A Suggestion Algorithm Instituted on Invasive Weed Optimization Algorithm and Bat Optimization Algorithm
}

\author{
Wisam Abdulelah Qasim, Ban Ahmed Mitras \\ Department of Mathematics, College of Computer Sciences \& Mathematics, Mosul, Iraq \\ Email: wesamalkwaaz@uomosul.edu.iq,Banah.mitras@uomosul.edu.iq
}

How to cite this paper: Qasim, W.A. and Mitras, B.A. (2020) A Suggestion Algorithm Instituted on Invasive Weed Optimization Algorithm and Bat Optimization Algorithm. Open Access Library Journal, 7: e6438. https://doi.org/10.4236/oalib.1106438

Received: May 17, 2020

Accepted: June 27, 2020

Published: June 30, 2020

Copyright (๑) 2020 by author(s) and Open Access Library Inc.

This work is licensed under the Creative Commons Attribution International License (CC BY 4.0).

http://creativecommons.org/licenses/by/4.0/

\section{(c) (i) Open Access}

\begin{abstract}
In this paper, two algorithms first, considered to be one of hybrid algorithms. And it is algorithm represents an Invasive Weed Optimization (IWO). This algorithm is a random numerical algorithm and the second algorithm representing the Bat Optimization Algorithm (BA). This algorithm is one of the swarm intelligence algorithms in smart optimization. The Invasive Weed Optimization Algorithm is inspired by nature, as the weeds have colonial behavior and were introduced in 2006 by Mehrabian and Lucas. Invasive weeds pose a serious threat to crops due to their adaptability and pose a threat to the overall planting cycle. In the Invasive Weed Optimization Algorithm, the behavior of these weeds was analyzed and implemented. The Bat algorithm, which is called a swarm intelligence algorithm, was used to achieve the goal and the best solution. The algorithm was designed by Yang in 2010 and taking advantage of the intelligence of the squadrons is to avoid falling into local solutions so the new hybridization process between the previous algorithms Invasive Weed Optimization (IWO) and Bat Optimization Algorithm (BA) and we will symbolize the new algorithm IWO-BA Comparing the hybrid algorithm suggested with the original algorithms the results were excellent. In most research functions, the optimal solution has been found.
\end{abstract}

\section{Subject Areas \\ Information, Communication Theory, Algorithms}

\section{Keywords}

Invasive Weeds Optimization Algorithm, Bat Optimization Algorithm, Hybrid Algorithms, Optimization 


\section{Introduction}

In our daily lives, the optimization is used to increase profits while reducing costs, maximize product performance while at the same time. Optimization was an active research field many decades ago and is still today. Recent years have seen the emergence of many complex optimization problems that are the reason why highly efficient algorithms are developed. There is currently a wide range of algorithms for many problems, and designers have worked to create or discover new algorithm, and new ideas developed in optimization and applications will therefore be urgently needed [1] [2].

One of the latest developments in the past two decades is the tendency used in the intuitive algorithms. Note that the algorithms (intuitive or post-intuitive) comprise the vast majority of modern optimisation techniques. We note that they have become very useful and effective in solving complex optimisation problems and have applied these algorithms in all important fields [3].

The particular algorithms represent the majority of the classical algorithms. A clear example of linear programming is the simplex method. It is a particular algorithm. They also consider the use of the derivative and are referred to as derivative algorithms. An example of this is the Newton-Raphson algorithm [2].

One of the random algorithmic methods is (Intuitive methods and Post-Intuitive methods). In 1986 Glover coined the word intuitive algorithm, which is the evolution of the intuitive algorithm [2]. The post-intuitive algorithm operates at a level higher than the intuitive algorithm. We remember that all post intuitives are used (Confirmed swap for random distribution and Local Search) [2].

It is noteworthy that there is no accepted description of methods (intuitive or post-intuitive) in which the term (intuitive and post-intuitive) is used interchangeably and that the recent trend refers to the classification of all random algorithms using intuitive algorithmic local analysis and random distribution [4].

The two important elements that are considered essential elements in the (post-intuitive) algorithm are (Intensification and Diversification) [4].

The post intuitive can be classified in several methods. Such approaches can be categorized according to the trajectory and society. The genetic algorithm is an example. The method of simulating steel is that it uses a single element which moves within the search space and the search method is a piece that is the best solution or the best way to move.

It is generally acceptable and when the step is not successful, it normally acknowledges a certain probability, as the movements affect the direction in the search space and the likelihood of non-zero, the path to a thorough optimization [4].

The research problem centered on finding the optimum comprehensive solution for the problems of unregulated and high measurement and avoiding falling into the problems of local solutions using the characteristics of the swarms algo- 
rithm, one of the algorithms of the swarms algorithm Bat (BA) was selected [5].

The purpose of the work is to develop a new way of solving the optimization problems, which is a difficult issue, in order to benefit the designers of the proposed hybrid algorithm and any topic falls within the difficult issues.

The goal of the research is to suggest a new algorithm called the Hybrid Algorithm where the algorithm (Invasive Weed Optimization) and the algorithm (Bat optimization) and the resulting IWO-BA algorithm is hybrid algorithms to take advantage of the positive qualities and reduce the negatives of the two previous algorithms.

Hybrid algorithm has been implemented in recent years, but programmers have used it since the 1980s. At present, we have exploited the combination of the various components of different research techniques and the tendency to design hybrid techniques used in artificial intelligence and operational research.

\section{Invasive Weed Optimization Algorithm (IWO)}

It is an algorithm that Mehrabian and Lucas first developed in 2006, 2006 and the algorithm is Behavior by biology. It is an algorithm for numerical optimization that actually simulates the natural behavior of weeds in colonization and finds a good location for growth and reproduction. Some of the characteristics of the IWO algorithm compared with other developmental algorithms, They are breeding methods, spatial dispersion and competitive exclusion [6].

\section{IWO algorithm includes multiple key steps are:}

\subsection{Steps of IWO}

Step 1 initial population

"A search area is taken and a number of weeds are initialized at random in the full search space"

Step 2 cloning

Plants produce seeds based on the role provided for fitness. As well as the (upper-lower) limits of the colony's dignity rule. We note that the number of seeds produced by the plant increases linearly from the minimum seed production possible to the maximum [6].

$$
\text { seed }_{i}=\text { floor }\left(\left(f_{i}-f_{\text {min }}\right) /\left(f_{\text {max }}-f_{\text {min }}\right)\left(S_{\text {max }}-S_{\text {min }}\right)\right)
$$

floor. Show that the seeds are rounded to the nearest whole number.

$f_{i}$ : The fitness function represents $i$ of weed.

$f_{\max }$ and $f_{\min }$ : The maximum and minimum value of the fitness function.

$S_{\min }$ and $S_{\max }$ : It represents the maximum and minimum number of seeds.

The above Equation (1) represents the relationship between the function value and the number of seeds of weeds if you increase the fitness function value the number of seeds varies between the max value and the min value [7].

Step 3 Spatial scattering

In this phase randomly generated seeds are distributed in the whole search space over dimensions. By normal zero-rate distribution and specific (variable) 
variance. This step means the seeds will be distributed randomly around the mother plant.

Nevertheless, at each step, the standard deviation of the random function will decrease from a predetermined initial value $\left(\sigma_{\text {initial }}\right)$ to a final value $\left(\sigma_{\text {final }}\right)$ at each step as:

$$
\sigma_{\text {iter }}=\left(\text { iter }_{\max }-i t e r\right)^{n} /\left(\text { iter }_{\max }\right)^{n}\left(\sigma_{\text {initial }}-\sigma_{\text {final }}\right)+\sigma_{\text {final }}
$$

$\sigma_{i t e r}:$ It represents the standard deviation in the current step.

iter $_{\max }$ : Represents the maximum number of iterations.

$n$ : Represents a non-linear transformation indicator.

To determine where the new seeds are located, we use the following equation:

$$
X_{\text {son }}=X_{\text {parent }}+s d=X_{\text {parent }}+\operatorname{randn}(0,1) * \sigma_{\text {iter }}
$$

Step 4 Spatial exclusion

Competition exists between plants for survival, and if the plant has no offspring and its fate is extinct when $P_{\max }$ exceeds the colony's maximum number of plants, the cycle of exclusion for a poor fitness plant will begin.

\subsection{Mechanism for Exclusion}

Once the maximum amount of herbs is reached in the colony, each herb will produce seeds and then spread to the research site. When all the seeds in their place are concentrated they are fitted with the mother plant (colony). Weeds with a low fitness function are excluded so as to achieve the maximum acceptable to the colonized community. Remember that plants and their offspring should survive and have a repetition in the algorithm as well as the item that has a high fitness function [6].

\section{Bat Optimization Algorithm (BA)}

Bat optimization algorithm is inspired by biology and is a heuristic method for solving difficult optimization problems. It represents an attempt to simulate the behavior of bats to hunt prey and the algorithm was presented by the research Yang in (2010) [8]. Bat Algorithm is based on the bat community, which is flying through the search solutions space in a specific or special order to find the best areas. Each bat element represents one solution in $(n)$ dimensions of the search space. The solutions are evaluated based on the appropriate value and using the given fitness function [9].

For example, the dimensions ( $n)$ are a real value for the optimization solution space. Each solution represents a bat, and evaluation is done using the given fitness function. There are also two real values of the dimension ( $n)$ of vectors associated with every bat in society [9].

The first vector is the value of the real value and represents the location of the bat in the solution to the search space.

The second vector is the real value vector and represents the velocity in all directions of dimensions $(\mathrm{n})$. 
The location and velocity of the vectors randomly configured at the beginning of the algorithm. The main link of the algorithm includes improved redundancy in the available solutions [9].

On each occurrence the appropriate value of each element in the bat group is calculated by the given fitness function. The new velocity vector is calculated on the basis of the relative distance from the best and current solution in society. Later, the position of all the bats is updated according to the speed vector. At the end of each iteration, the best solution is created and used as a new reference point, and the search space continues to be explored until the stopping condition is met. Usually, this condition is the maximum number of iterations or improvements in the best solution [9].

\subsection{Bat Motion}

First, we have to define the laws of the location of the bat $\left(X_{i}\right)$ and their velocity $\left(V_{i}\right)$ in the dimensions $(d)$ of the search space as new solutions $\left(X_{i t}\right)$ and velocity $\left(V_{i t}\right)$ are updated at the time of step $(t)$ using the following equations:

$$
\begin{gathered}
f_{i}=f_{\text {min }}+\left(f_{\text {max }}-f_{\min }\right) \beta \\
V_{i t+1}=V_{i t}+\left(X_{i t}-X^{*}\right) f_{i} \\
X_{i t+1}=X_{i t}+V_{i t}
\end{gathered}
$$

As-:

$\beta$ : represents a random vector taken from a uniform distribution

$X^{*}$ : represents the best comprehensive local site obtained after comparing

$f_{\max }$ : represents the highest value in frequency and pulse emission.

$f_{\min }$ : represents the lowest frequency and pulse emission value.

Depending on the field size of the problem, each bat is random frequency, which exists uniformly from $\left\{f_{\min }, f_{\max }\right\}$ as the local search portion represents one of the selected solutions among the best current solutions. A new solution to each locally generated bat is by using random walk behavior. As in the equation.

$$
X_{\text {new }}=X_{\text {old }}+\varepsilon A_{t}
$$

$\varepsilon$ is a random vector between $[-1,1]$

$\left\langle A_{i t}\right\rangle=A_{t}$ : represents the loud rate for all bats in the time step.

This is similar to updating bat velocities and locations for some actions in improving standard particle swarm, as $f_{i}$ basically controls the speed and range of motion of aggregate particles. To some extent, the bat algorithm (BA) can be seen as a balanced mix of optimization of particle swarm optimization and intense local search controlled by sound and pulse rate [8].

\subsection{Real Echolocation Behavior of Bats}

One of the creations of animal life studied by many zoologists is the echo-locating (or biological sonar) of bats [10]. There are a few other animal groups that also have echo-positioning capabilities such as birds (South American oilbirds and 
south-east Asian swiftelts), whales, dolphins, and small insects, but this is very rare. This behavior of bats began by Lazzaro Spallanzani in (1794). Then the term "echolocation" was introduced by Donald Griffin in 1944 with the ability of bats to produce sound with echoes beyond the frequency of human hearing and using it for general guidance in the dark and finding prey at night with echo location, bats emit ultrasound pulses with either a modified frequency (FM) or fixed frequency (CF) and sometimes a mixture of the two [11] [12]. Tonal signals produced in the larynx (some bats use the tongue) are emitted in short bursts through the mouth or gills [13]. Suga in (1990) described that the reflected sounds were in a state of Doppler pressure or transformation which made the received resonance at a higher frequency than previously produced sound. Bats can determine the object and distance by measuring the modified echo reflection time [12] [14].

When bats begin to search for prey in the research phase, they emit pulses at a low rate around the $10 \mathrm{~Hz}$ frequency [13]. During the approach phase, where bats discover and approach prey, the pulses must be shorter to prevent interference [14]. The shorter pulses cause a decrease in time between the pulse and echo. Also at this moment, the pulse emission rate gradually increases to 200 beats per second as bats continue to update the prey site [14]. Suga (1990) states that the pulse emission rate increases because bats need to produce more signals to accurately follow prey as the angular position of prey changes more quickly due to the closer distance between the bat and the object. In the last stage (final stage), the frequency of the emitted pulses increases by more than $200 \mathrm{~Hz}$ and the pulse emission rate becomes faster in only a small fraction of a millisecond long before the prey is caught [13]. A colony of bats has two exclusive approaches to avoid colliding with one another while echo locating [15]. This behavior mostly applies to vampire bat types such as regurgitation from blood meals from successful bats to feed them to their useless member in the colony as a response to the meticulously balanced energy budget of each member of the colony [16]. Wilkiuson discovered (1988) that surviving altruistic behavior grows in survivors so that his fitness is relatively high to non-recipient, and mutual altruism also occurs during community nursing [17].

\subsection{Step Bat Algorithm}

Objective function $f(x), \quad x=\left(x_{i}, \cdots, x_{d}\right)^{\mathrm{T}}$

Initialize the bat population $X_{i}(i=1,2, \cdots, n)$ and $V_{i}$

Define pulse frequency $f_{i}$ at $X_{i}$

Initialize pulse rates $r_{i}$ and the loudness $A_{i}$

While ( $t<$ Max number of iterations)

Generate new solutions by adjusting the frequency,

And updating velocities and location/solutions (Equations (1) to (3))

If $\left(\right.$ rand $>r_{i}$ )

Select a solution among the best solutions

Generate a local solution around the selected best solution 
end if

Generate a new solution by flying randomly

if $\left(\right.$ rand $\left.<A_{i} \& f\left(X_{i}\right)<f\left(X^{*}\right)\right)$

Accept the new solutions

Increase $r_{i}$ and reduce $A_{i}$

end if

Rank the bats and find the current best $x^{*}$

end while

post process results and visualization [18].

\section{Hybrid Algorithm}

Several researchers built algorithms by hybridizing common and difficult problems with the NP-Hard. Researchers have therefore worked in recent years to hybridize algorithms with normal and difficult problems and have achieved the best results by applying hybrid problems. The real purpose of the hybridization process is to obtain general and varied solutions that can deal with problems in the real world. Hybrid algorithms have focused on swarm intelligence, a recently recognized branch of artificial intelligence that studies complex self-regulating and decentralized systems' collective behaviours. In 1989, the term swarm intelligence emerged as a set of algorithms by the two researchers Jingwing and Geradobeni and was used to control robot swarms [1].

In addition to the IWO optimization algorithm to BA algorithm, a detailed solution can be found for optimization problems.

\section{Hybrid Invasive Weed Algorithm}

The new hybrid algorithm has been named IWO-BA proposed. The Algorithm for Invasive Weed Optimization is different from other evolutionary algorithms including: (reproduction, spatial dispersion and competitive exclusion) The optimum gain from the application of these properties in the hybridization process was achieved.

\section{Practical Aspect}

The hybrid algorithm (IWO-BA) has been tested in terms of efficiency by using this algorithm to solve 10 a function of numerical optimization functions which is a highly efficient problems.

The IWO-BA hybrid algorithm is a combination of the algorithms for Invasive Weed optimization and the algorithm of BAT.

When comparing the three algorithms, we notice that the invasive weed algorithm gives far results and the bat algorithm gives spaced results, but when performing the hybridization process between the two algorithms, we note the improvement of the results for most functions.

Tables 1-6 below shows the initial parameters that must be specified before starting the program. 
The test was carried out using a computer with the following specifications: CPU speed is $2.4 \mathrm{GHz}$.

RAM size is $4 \mathrm{~GB}$

The Matlab R2013a program works on windows 7.

Table 1. Initial parameters.

\begin{tabular}{cccc}
\hline Parameter & IWO & BA & IWO-BA \\
\hline Npop0 & $5-10-15-20$ & $5-10-15-20$ & $5-10-15-20$ \\
Npop & 25 & 25 & 25 \\
N & 2 & --- & 2 \\
Smin & 0 & --- & 0 \\
Smax & 5 & --- & 5 \\
Maxit & 1000 & 1000 & 1000 \\
Sigma_initial & 0.5 & --- & 0.5 \\
Sigma_final & 0.001 & --- & 0.001 \\
\hline
\end{tabular}

Table 2. Test functions.

$F_{1}(x)=\sum_{i=1}^{n} x_{i}^{2}$
$F_{2}(x)=\sum_{i=1}^{n}\left|x_{i}\right|+\prod_{i=1}^{n}\left|x_{i}\right|$
$F_{3}(x)=\sum_{i=1}^{n}\left(\sum_{j-1}^{i} x_{i}\right)^{2}$
$F_{4}(x)=\max _{i}\left\{\left|x_{i}\right|, 1 \leq i \leq n\right\}$
$F_{5}(x)=\sum_{i=1}^{n}\left|x_{i}^{2}-10 \cos \left(2 \pi x_{i}\right)+10\right|$
$F_{6}(x)=-20 \exp \left(-0.2 \sqrt{\frac{1}{n} \sum_{i=1}^{n} x_{i}^{2}}\right)-\exp \left(\frac{1}{n} \sum_{i=1}^{n} \cos \left(2 \pi x_{i}\right)\right)+20+e$
$F_{7}(x)=\frac{1}{4000} \sum_{i=1}^{n} x_{i}^{2}-\prod_{i=1}^{n} \cos \left(\frac{x_{i}}{\sqrt{i}}\right)-1$
$F_{9}(x)=\frac{\pi}{n}(x)=4 x_{i=1}^{2}-2.1 x_{1}^{4}+\frac{1}{3} x_{1}^{6}+x_{1} x_{2}-4 x_{i}^{2}+\frac{x_{i}\left(b_{i}^{2}+b_{i} x_{2}\right)}{b_{i}^{2}+b_{i} x_{2}+x_{4} \mid}$
$y_{i}=1+\frac{X_{i+1}}{4} u\left(x_{i}, a, k, m\right)=\left\{\begin{array}{l}\left.\left.1+10 \sin ^{2}\left(\pi y_{i+1}\right)\right]+\left(y_{n-1}\right)^{2}+\sum_{i=1}^{n} U\left(x_{i}, 10,100,4\right)\right) \\ k\left(x_{i}-a\right)^{m} x_{i}>0 \\ o\left(-a x_{i}<a\right.\end{array}\right.$


Table 3. The results of the algorithm IWO and BA and compared with the results of hybrid algorithm IWO-BA by using 5 elements and 1000 iterations.

\begin{tabular}{cccc}
\hline Function & IWO & BA & IWO-BA \\
\hline$F_{1}$ & $3.48348 \times 10^{-5}$ & 2.3392 & $5.0085 \times 10^{-204}$ \\
$F_{2}$ & 0.1421731 & 3.0180 & $3.8296 \times 10^{-102}$ \\
$F_{3}$ & $3.847 \times 10^{-4}$ & 1.5992 & $3.4584 \times 10^{-204}$ \\
$F_{4}$ & 31.06199 & 0.7961 & $1.3826 \times 10^{-102}$ \\
$F_{5}$ & 121.29128 & 13.9298 & 0 \\
$F_{6}$ & 19.10598 & 2.9917 & $0.8818 \times 10^{-16}$ \\
$F_{7}$ & $9.8396308 \times 10^{-3}$ & 0.1364 & 0 \\
$F_{8}$ & 25.26338 & $2.9402 \times 10^{-12}$ & 0.53027 \\
$F_{9}$ & $3.9978 \times 10^{-4}$ & 0.0058 & 0.020363 \\
$F_{10}$ & -1.0316 & -1.0316 & -1.0316 \\
\hline
\end{tabular}

Table 4. The results of the algorithm IWO and BA and compared with the results of hybrid algorithm IWO-BA by using 10 elements and 1000 iterations.

\begin{tabular}{cccc}
\hline Function & IWO & BA & IWO-BA \\
\hline$F_{1}$ & $3.38112 \times 10^{-5}$ & 0.4724 & $7.459 \times 10^{-204}$ \\
$F_{2}$ & 0.0247183 & 3.1961 & $6.5138 \times 10^{-102}$ \\
$F_{3}$ & $1.91002 \times 10^{-3}$ & 0.2387 & $1.5755 \times 10^{-203}$ \\
$F_{4}$ & 32.73155 & 0.5602 & $1.0758 \times 10^{-102}$ \\
$F_{5}$ & 85.77135 & 12.9348 & 0 \\
$F_{6}$ & 19.1387 & 3.0271 & $0.8818 \times 10^{-16}$ \\
$F_{7}$ & $8.865911 \times 10^{-2}$ & 0.0037 & 0 \\
$F_{8}$ & 19.86204 & $3.6987 \times 10^{-15}$ & 2.6507 \\
$F_{9}$ & $2.45339 \times 10^{-3}$ & 0.0114 & 0.00068788 \\
$F_{10}$ & -1.0316 & -1.0316 & -1.0316 \\
\hline
\end{tabular}

Table 5. The results of the algorithm IWO and BA and compared with the results of hybrid algorithm IWO-BA by using 15 elements and 1000 iterations.

\begin{tabular}{cccc}
\hline Function & IWO & BA & IWO-BA \\
\hline$F_{1}$ & $3.31392 \times 10^{-5}$ & $1.3777 \times 10^{-06}$ & $5.0022 \times 10^{-204}$ \\
$F_{2}$ & 0.0252103 & 0.2212 & $5.043 \times 10^{-102}$ \\
$F_{3}$ & $1.786933 \times 10^{-4}$ & 0.3076 & $6.3264 \times 10^{-204}$ \\
$F_{4}$ & $2.62742 \times 10^{-3}$ & 0.3570 & $6.8396 \times 10^{-103}$ \\
$F_{5}$ & 76.22018 & 20.8943 & 0 \\
$F_{6}$ & 19.07697 & 3.0266 & $8.8818 \times 10^{-16}$ \\
$F_{7}$ & $1.377530 \times 10^{-2}$ & $1.1428 \times 10^{-07}$ & 0 \\
$F_{8}$ & 18.07077 & $1.9280 \times 10^{-14}$ & 0.0020668 \\
$F_{9}$ & $7.67408 \times 10^{-4}$ & $4.3834 \times 10^{-04}$ & 0.00082957 \\
$F_{10}$ & -1.0316 & -1.0316 & -1.0316 \\
\hline
\end{tabular}


Table 6. The results of the algorithm IWO and BA and compared with the results of hybrid algorithm IWO-BA by using 20 elements and 1000 iterations.

\begin{tabular}{cccc}
\hline Function & IWO & BA & IWO-BA \\
\hline$F_{1}$ & $3.31477 \times 10^{-5}$ & $1.0246 \times 10^{-06}$ & $4.5128 \times 10^{-204}$ \\
$F_{2}$ & 0.026135 & 0.0812 & $5.5131 \times 10^{-102}$ \\
$F_{3}$ & $1.367628 \times 10^{-3}$ & $2.0651 \times 10^{-06}$ & $1.0342 \times 10^{-203}$ \\
$F_{4}$ & $2.48269 \times 10^{-3}$ & 0.0705 & $1.3485 \times 10^{-16}$ \\
$F_{5}$ & 89.4533 & 3.9801 & 0 \\
$F_{6}$ & 18.8478 & 2.3169 & $0.8818 \times 10^{-16}$ \\
$F_{7}$ & $1.747331 \times 10^{-2}$ & $1.8657 \times 10^{-07}$ & 0 \\
$F_{8}$ & 18.44998 & $2.0395 \times 10^{-13}$ & 0.011654 \\
$F_{9}$ & $4.7212 \times 10^{-4}$ & 0.0059 & 0.00071409 \\
$F_{10}$ & -1.0316 & -1.0316 & -1.0316 \\
\hline
\end{tabular}

\section{Conclusion}

In this study, we researched the Invasive Weed Optimization Algorithm and we saw poor performance. This algorithm in terms of reaching the local Micropoint indicates a divergence from the optimal solution and improving the performance of this algorithm and avoiding falling into local solutions that we hybridized with swarm algorithms with swarm characteristics and the algorithm is a Bat optimization algorithm and the hybrid algorithm IWO-BA is the new algorithm proposed, at the start of the IWO-BA hybrid algorithm, we compared the results of the IWO and BA algorithms. We got very good results. For most (10) test functions the optimum overall solution has been achieved. Our results indicate this.

\section{Conflicts of Interest}

The authors declare no conflicts of interest regarding the publication of this paper.

\section{References}

[1] Parsopoulos, K.E. and Vrahatis, M.N. (2010) Particle Swarm Optimization and Intelligence: Advances and Applications. IGI Global, Hershey, PA.

https://doi.org/10.4018/978-1-61520-666-7

[2] Yaseen, H.T., Mitras, B.A. and Khidhir, A.S.M. (2018) Hybrid Invasive Weed Optimization Algorithm with Chicken Swarm Optimization Algorithm to Solve Global Optimization Problems. International Journal of Computer Networks and Communications Security, 6, 173-181.

[3] Yang, X.-S. (2010) Engineering Optimization: An Introduction with Metaheuristic Applications. John Wiley \& Sons, Hoboken. https://doi.org/10.1002/9780470640425

[4] Yang, X.-S. (2010) Nature-Inspired Metaheuristic Algorithms. Luniver Press, Frome.

[5] Blum, C., Roli, A. and Sampels, M. (2008) Hybrid Metaheuristics: An Emerging Approach to Optimization. Vol. 114, Springer, Berlin. 
https://doi.org/10.1007/978-3-540-78295-7

[6] Mehrabian, A.R. and Lucas, C. (2006) A Novel Numerical Optimization Algorithm Inspired from Weed Colonization. Ecological Informatics, 1, 355-366.

https://doi.org/10.1016/j.ecoinf.2006.07.003

[7] Zhao, Y., Leng, L., Qian, Z. and Wang, W. (2016) A Discrete Hybrid Invasive Weed Optimization Algorithm for the Capacitated Vehicle Routing Problem. Procedia Computer Science, 91, 978-987. https://doi.org/10.1016/j.procs.2016.07.127

[8] Yang, X.S. (2011) Bat Algorithm for Multi-Objective Optimisation. International Journal of Bio-Inspired Computation, 3, 267-274. https://doi.org/10.1504/IJBIC.2011.042259

[9] Kiełkowicz, K. and Grela, D. (2016) Modified Bat Algorithm for Nonlinear Optimization. IJCSNS International Journal of Computer Science and Network Security, $16,46-50$.

[10] Simmons, J.A., Howell, D.J. and Suga, N. (1975) Information Content of Bat Sonar Echoes: Recent Research on Echolocation in Bats Identifies Some of the Kinds of Information Conveyed by Echoes of Their Sonar Sounds. American Scientist, 63, 204-215.

[11] Surlykke, A., Futtrup, V. and Tougaard, J. (2003) Prey-Capture Success Revealed by Echolocation Signals in Pipistrelle Bats (Pipistrellus pygmaeus). Journal of Experimental Biology, 206, 93-104. https://doi.org/10.1242/jeb.00049

[12] Ghose, K., Horiuchi, T.K., Krishnaprasad, P.S. and Moss, C.F. (2006) Echolocating Bats Use a Nearly Time-Optimal Strategy to Intercept Prey. PLoS Biology, 4, e108. https://doi.org/10.1371/journal.pbio.0040108

[13] Altringham, J.D., Hammond, L. and McOwat, T. (1996) Bats: Biology and Behaviour. Vol. 3, Oxford University Press, Oxford.

[14] Suga, N. (1990) Biosonar and Neural Computation in Bats. Scientific American, 262, 60-68. https://doi.org/10.1038/scientificamerican0690-60

[15] Vogler, B. and Neuweiler, G. (1983) Echolocation in the Noctule (Nyctalus noctula) and Horseshoe Bat (Rhinolophus ferrumequinum). Journal of Comparative Physiology A: Neuroethology, Sensory, Neural, and Behavioral Physiology, 152, 421-432. https://doi.org/10.1007/BF00606247

[16] Denault, L.K. and McFarlane, D.A. (1995) Reciprocal Altruism between Male Vampire Bats, Desmodus rotundus. Animal Behaviour, 49, 855-856.

[17] Oliver, J. (2013) Improved Docking of Polypeptides with Glide. Journal of Chemical Information and Modeling, 53, 1689-1699. https://doi.org/10.1021/ci400128m

[18] Yang, X.S. (2010) A New Metaheuristic Bat-Inspired Algorithm. Studies in Computational Intelligence, 284, 65-74. https://doi.org/10.1007/978-3-642-12538-6_6 